\title{
Guest Editorial: Special Section on Modern Time-Frequency Analysis
}

\author{
Bingo Wing-Kuen Ling ${ }^{1}$
}

Published online: 7 June 2018

CC Springer Science+Business Media, LLC, part of Springer Nature 2018

Welcome to this Special Section on modern time-frequency analysis. The time-frequency analysis finds many applications in various disciplines of science and engineering. For example, the wavelet is used in the JPEG2000 standard for performing the image compression. The empirical mode decomposition and the singular spectral analysis are used to extract the underlying trend of the financial signal for performing the data prediction. The wavelet, the empirical mode decomposition and the singular spectral analysis are widely used for performing signal denoising and feature extraction in various pattern recognition applications. The filter bank is used in the orthogonal frequency division multiplexing in the $5 \mathrm{G}$ communication.

Although the time-frequency analysis has been extensively studied for nearly 50 years, there are still many unsolved problems. For example, in the filter bank theory, the conditions for general high-dimensional nonuniform filter banks achieving exact perfect reconstruction are unknown. For the empirical mode decomposition, the set of signals that can be decomposed is unknown. Even though the empirical mode decomposition can be performed, the conditions for the mode mixing phenomenon are unknown. For the singular spectral analysis, the relationship between the center frequencies of the decomposed components and the eigenvalues of the trajectory matrix is unknown. The main objective of this Special Section is to provide our readers with a focused set of peer-reviewed articles that address these problems and reflect some recent advances in the modern time-frequency analysis. It also serves as a forum for

$\bowtie \quad$ Bingo Wing-Kuen Ling yongquanling@gdut.edu.cn

1 School of Information Engineering, Guangdong University of Technology, No. 100, Waihuan Xi Road, Guangzhou University Mega Center, Guangzhou City 510006, China 
the researchers to exchange their latest findings. We hope that it will stimulate further research and development in the area of modern time-frequency analysis.

More than twenty papers were submitted to this Special Section, covering both the practical and theoretical aspects. After a rigorous peer review process, only eleven papers have been selected. The following is a brief summary of the selected papers.

The article "Exploiting temporal correlation for detection of non-stationary signals using a de-chirping method based on time-frequency analysis," by Nabeel Ali Khan and Sadiq Ali, proposes a method for the detection of non-stationary signals in the presence of noise with uncertain power. The method uses instantaneous frequency estimation and de-chirping procedure to convert a non-stationary signal into a stationary signal, thus allowing one to exploit temporal correlation as an extra feature for signal detection in addition to the signal energy. The proposed method can be used for both the mono-sensor and the multi-sensor recording. Simulation results indicate the superiority of the proposed approach.

The article "Locally optimized adaptive directional time-frequency distributions," by Mokhtar Mohammadi, Ali. A. Pouyan, Nabeel Ali Khan and Vahid Abolghasemi, is concerned with the estimation of the parameters of adaptive directional time-frequency distributions. Adaptive directional time-frequency distributions locally optimize the direction of the smoothing kernel on the basis of directional Gaussian or double derivative directional Gaussian filter. Conventionally, the parameters have to be tuned manually for each particular signal. When the signal encompasses close or shortduration components, the global optimal solution of the parameter estimation problem fails to provide the desired results. This paper proposes a two-stage algorithm to resolve this issue. It is shown that the multistage algorithm can result in a time-frequency distribution that has both high resolution for the close components and a good concentration of the signal energy for the short-duration signal components. The experimental findings reveal the superiority of the proposed technique over the existing methods.

The paper "Reconstruction of non-stationary signals with missing samples using time-frequency filtering," by Nabeel Ali Khan and Mokhtar Mohammadi, proposes a time-frequency method for recovering missing samples from multi-component signals. This is achieved by a combination of a sparsity aware time-frequency signal analysis method and time-frequency filtering technique. The sparsity aware time-frequency method overcomes the distortions caused by the missing samples in the time-frequency domain. This is followed by time-frequency filtering techniques for recovering the signals. All the extracted components are then combined to recover the complete signal. The proposed method outperforms other signal recovery methods such as the gradient descent algorithm and the matching pursuit.

The article "Using the Lambert-W function to create a new class of warped time-frequency representations," by Amal Feltane, G. Faye Boudreaux-Bartels and Yacine Boudria, proposes a warping function using the Lambert $\mathrm{W}$ function to create a class of warped time-frequency representations. Some examples are provided to demonstrate how the warping function can be used on a variety of nonlinear frequencymodulated chirp signals to linearize their support in the warped time-frequency plane. An algorithm has been proposed to optimize the parameter of the warping function.

The article "A complex-valued mixing matrix estimation algorithm for underdetermined blind source separation," by Qiang Guo, Guoqing Ruan and Liangang Qi, 
considers complex-valued mixing matrix estimation in underdetermined blind source separation. An estimation algorithm based on both single source point detection and modified dynamic data field clustering has been proposed. Simulation results show that the proposed method can estimate the complex-valued mixing matrices with high accuracy, especially in the real-world noncooperative cases without the prior knowledge. These results are useful for performing underdetermined blind source separation.

The article "Nonuniform sampling theorems for bandlimited signals in the offset linear canonical transform," by Xu Shuiqing, Huang Lei, Chai Yi and He Yigang, addresses the problem of nonuniform sampling of deterministic signals and random signals in the offset linear canonical transform domain. First, the authors introduce some special nonuniform sampling models. Then, they derive reconstruction theorems for the deterministic signals from these nonuniform samples in the offset linear canonical transform domain. In addition, the nonuniform sampling theorems for random signals in the offset linear canonical transform domain have also been derived. Finally, simulation results are presented to show the advantages and effectiveness of the methods.

The article "Epoch estimation from emotional speech signals using variational mode decomposition," by G. Jyothish Lal, E. A. Gopalakrishnan and D. Govind, presents a novel approach for estimating epochs of the emotional speech signal, epochs being the time locations where a significant excitation occurs in the vocal tract during the production of the voiced sound by the vibration of vocal folds. The estimation of the epoch locations is essential for deriving the instantaneous pitch contours for performing accurate emotion analysis. The proposed approach exploits the effectiveness of an adaptive time series decomposition technique called the variational mode decomposition for estimating the epochs. The performance of the proposed method has been evaluated using six different emotions taken from the German emotional speech database with simultaneous ECG signals. Experimental results on clean emotive speech signals show that the proposed method provides an identification rate and an accuracy comparable to that of the best existing algorithm, in addition to providing a better reliability for performing epoch estimation of emotive speech signals degraded by the presence of noise.

The article "A new hybrid active noise control system with convex combination of time and frequency domain filtered-X LMS algorithms," by Trideba Padhi, Mahesh Chandra, Asutosh Kar and M.N.S. Swamy, proposes a hybrid active noise control system using a convex combination of the time and frequency domain filtered-X LMS algorithms. The feed forward part uses a time domain filtered-X LMS algorithm to control the disturbances that are correlated with the reference noise. On the other hand, the feedback part uses a multi-resolution analysis-based frequency domain wavelet packet filtered-X LMS algorithm to control the uncorrelated disturbances encountered during the operation of an active noise control system. An extra adaptive filter is used to smoothen the error signal in the hybrid active noise control system. As a result of the convex combination of the time domain filtered-X LMS and the wavelet packet filtered-X LMS algorithms in a hybrid active noise control system, the proposed method is successful for canceling the disturbances encountered by the active noise control system from multiple noise sources. 
The article "Design of time-frequency localized two-band orthogonal wavelet filter banks," by Dinesh Bhati, Ram Bilas Pachori, Manish Sharma and Vikram M. Gadre, deals with the design of time-frequency localized two-band orthogonal wavelet filter banks using convex semidefinite programming. The sum of the time and frequency variances of the filter is used to formulate a real symmetric positive definite matrix in order to achieve the joint time-frequency localized filter. For the non-maximally regular two-band filter banks with the lengths equal to twenty, the authors find that the solution of the semidefinite programming converges to the filters with the time-frequency products almost the same as the Daubechies maximally regular filters with lengths equal to twenty when the regularity order is increased. Unlike the class of the Daubechies maximally regular minimum-phase wavelet filter banks, a rank minimization algorithm in a semidefinite programming is employed to obtain the mixed-phase low-pass filters with the time-frequency products of the filters as well as the scaling and wavelet functions better than the equivalent two-band Daubechies filter banks.

The article "Eigenvalue decomposition of Hankel matrix based time-frequency representation for complex signals," by Rishi Raj Sharma and Ram Bilas Pachori, proposes a time-frequency representation-based data-driven technique for the analysis of the complex-valued signals. The positive and the negative frequency components of the complex signals are separately decomposed using the recently developed Hankel matrix eigenvalue decomposition-based method. Furthermore, the Hilbert transform is applied to the decomposed components to obtain the time-frequency representation of both the positive and the negative frequency ranges. The proposed method for obtaining the time-frequency representation is compared to the existing methods. The results on the synthetic and the natural complex signals show that the proposed method performs better than the compared methods.

The article "Improved eigenvalue decomposition based approach for reducing cross-terms in Wigner-Ville distribution," by Rishi Raj Sharma and Ram Bilas Pachori, proposes a data-driven method to reduce the cross-terms in the Wigner-Ville distribution using improved eigenvalue decomposition of the Hankel matrix. This method decomposes a multi-component non-stationary signal into several mono-component non-stationary signals. Then, the amplitude-based segmentation is applied to obtain the components which are separated in the time domain. Further, frequency modulation rates of the components are observed to obtain an adaptive window. The adaptive window removes the intra-cross-terms which are generated due to the nonlinearity in the frequency modulation. Finally, the sum of the Wigner-Ville distributions of all the components is considered as the Wigner-Ville distribution of the multi-component non-stationary signal. The simulation study carried out on synthetic and natural signals shows the effectiveness of the proposed method. The performance of the proposed method has also been evaluated in additive white Gaussian noise environment.

The Guest Editor would like to thank all the authors for their contributions and express his deepest gratitude to the reviewers for their timely and high-quality reviews and for their effort in providing valuable feedback to the authors. Without their help, this Special Section would not have materialized. The Guest Editor would also like express his sincere appreciation to the Editor-in-Chief, Professor M.N.S. Swamy, who 
provided an opportunity to develop this Special Section, and for his constant guidance and support.

Sincerely, Bing Wing Kuen Ling

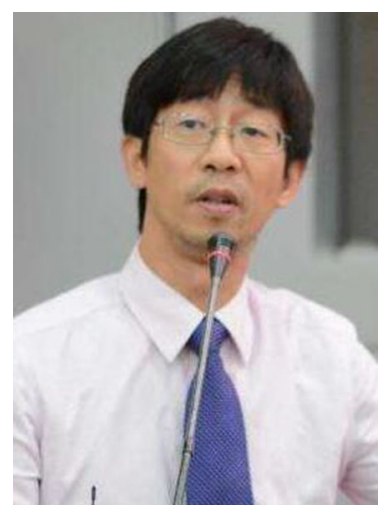

Bing Wing Kuen Ling (SM'98) obtained his B. Eng. and M. Phil. degrees from the Hong Kong University of Science and Technology in 1997 and 2000, respectively, and the Ph. D. degree from the Hong Kong Polytechnic University in 2003. He joined the King's College London as a lecturer between 2004 and 2010. Then, he joined the University of Lincoln as a Principal Lecturer in 2010 and then promoted to a Reader in 2011. In 2012, he joined the Guangdong University of Technology as a Hundred People Plan Distinguished Professor. He received the China Young Thousand People Plan Distinguished Professor Award in 2013, the IET Fellowship in 2013, and the Guangdong Province Pearl Scholar Award in 2015. He was an associated editor of the International Journal of Bifurcation and Chaos. He is currently an associate editor of the Journal of Franklin Institute, Circuits Systems and Signal Processing, Measurement, Journal of Industrial and Management Optimization, etc. He has organized 15 international conferences, such as the IEEE ICCE-C 2014, IEEE ICCE-C 2015, IEEE ICCE-C 2016, and 17 special sessions in various international conferences. His research interests include optimization theory, time-frequency analysis, biomedical signal processing, multimedia signal processing, nonlinear digital signal processing systems and control theory. He has published over 130 international journal papers and 120 international conference papers in these areas. 